\title{
Clinical Immersion Program for Bioengineering and Medical Students
}

\section{Prof. Susan Stirling, UIC School of Design, University of Illinois at Chicago}

Susan Stirling is a designer, researcher and educator. She has an undergraduate degree from the University of Wisconsin-Madison and a graduate degree from the Institute of Design at the Illinois Institute of Technology.

At the University of Illinois at Chicago she teaches Design Research Methods, Human Experience in Design and Interdisciplinary Product Development. Susan collaborates with non-design faculty to teach the design process, and helps students discover opportunities and solve problems with design. She is the co-instructor of the Clinical Immersion program in the Department of BioEngineering. Susan balances teaching with her professional career as a design researcher, consultant and strategist.

\section{Dr. Miiri Kotche, University of Illinois, Chicago}

Miiri Kotche is a Clinical Associate Professor of Bioengineering at the University of Illinois at Chicago, and currently serves as Director of the Medical Accelerator for Devices Laboratory (MAD Lab) at the UIC Innovation Center. Prior to joining the faculty at UIC, she worked in new product development for medical devices, telecommunications and consumer products. She co-teaches both bioengineering capstone design courses, including the longstanding core senior design sequence and the recently launched interdisciplinary medical product development course. She also serves as co-Director of the Freshman Engineering Success Program, and is actively involved in engineering outreach for global health. Miiri received her Ph.D. in Bioengineering and M.S. in Mechanical Engineering from the University of Illinois at Chicago and a B.S. in General Engineering from the University of Illinois at Urbana Champaign. 


\section{Clinical Immersion Internship Introduces Students to Needs Assessment}

\section{Abstract}

A summer Clinical Immersion experience for rising bioengineering seniors who are enrolled in a twosemester capstone design sequence is offered to provide exposure to the clinical environment, including process flow, workarounds, and a first-hand understanding of where and how medical devices are used. In 2016 the program was expanded to include rising second year medical students. The primary learning objective is to formalize a methodical approach to needs assessment based on user-centered design. In this six-week internship, students rotate 30 hours per week in a clinical department under a physician mentor. Students also receive weekly workshop instruction on user-centered design, empathy, contextual inquiry and stakeholder interviewing, analysis and synthesis of research, and prioritization of user needs. Students work in teams made up of 2 medical students and 2 rising senior bioengineering students and rotate through three-week long clinical rotations in two of the following departments: Anesthesia, Emergency Medicine, Gastroenterology, Hematology and Radiation Oncology, Neurology, Ophthalmology, Orthopedics, Pulmonary Critical Care, Radiology, and Urology. At the end of each rotation, teams present problem statements based on insights from their primary research as well as potential design concepts to address the identified needs. Participants maintain a blog to capture and reflect on their observations, which also allows them to share their experience with the other students. Upon completion of the Clinical Immersion program, Bioengineering students are well prepared for the senior design capstone course that emphasizes development of medical devices conceived from validated end-user needs. Select medical students, as part of their Innovation Medicine program, gain experience in interdisciplinary collaboration with engineers, exposure to the user-center process, and enhance their interpersonal skills. This paper also discusses implementation challenges and program modifications for Summer 2017.

\section{Introduction}

Closing the gap between the clinical environment and engineering design to ensure a user-centered focus in addressing the underlying problems that physicians, nurses and other stakeholders experience has been a greater priority in biomedical engineering education [5-10] in recent years. This paper is a continuation of the progress from the first three years of the Clinical Immersion Program beginning Summer 2014, including changes implemented in Summer 2016 as well as further modifications planned for the 2017 Program [1]. Interest in this program has been strong and grows every year demonstrated by the increase in applications to the program from bioengineering students. Feedback from past participants (see section 4) support the value of experiential learning which introduces students to clinical end users and teaches students valuable industry skill sets previously only available as "on the job training."

The Clinical Immersion summer program provides a valuable opportunity for students to observe and interact with clinicians in their work environment to better enable them to methodically identify opportunities and requirements. This experience helps them avoid the product gap [2,3], resulting from a failure to fully understand the user's needs. Immersion internships are important because they enable students to learn and apply user-centered design principles $[3,4]$. Rising undergraduate seniors who 
participate in the Clinical Immersion program the summer before enrolling in either the two-semester Senior Design capstone Interdisciplinary Medical Product Design ("IMPD") or the "traditional" senior design sequence are better prepared to efficiently and effectively translate user needs into product concepts and design requirements. Select medical students, who are part of the Innovation Medicine "IMED" co-curricular program, have the opportunity to work collaboratively with bioengineering students to identify problems for further research opportunities. The Clinical Immersion program (CIP) allows these medical students an interdisciplinary experience as part of the IMED curriculum.

Since 2011, the Department of Bioengineering is jointly operated within both the College of Medicine $(\mathrm{COM})$ and the College of Engineering. We are able to leverage our position within the COM to place students in a wide variety of clinical environments with full medical faculty support and engagement. The University of Illinois at Chicago operates the largest public medical school in the country, and distinguishes itself by its ability to provide medical students with clinical experiences early in their education. Bioengineering students and IMED medical students are teamed together to conduct a needs assessment in a clinical department. This direct exposure to the hospital, in addition to formal instruction on the use of methodical approaches to observe, interview, and identify and prioritize user needs, will better prepare students to identify both potential opportunities, but also better understand the requirements and constraints imposed by users and their environment.

\section{Program Structure}

\section{Clinical Immersion Program Details 2016}

The Clinical Immersion program has expanded and evolved in the three years of operation based on feedback from students and clinical mentors. In 2014, bioengineering students rotated in pairs in (2) three-week long rotations in the following departments: Anesthesiology, Gastroenterology/Hepatology, Hematology/Oncology, Ophthalmology, Orthopedics, or Transplant Surgery. In 2015, the program expanded to include Pulmonary Critical Care, Radiology and Urology. Details about the initial program structure and implementation of the Clinical Immersion Program have been discussed previously [1]. The program is a paid student internship for Bioengineering students at The University of Illinois at Chicago. Each Monday morning, participants spent three hours in workshop with the two program instructors, from the Department of Bioengineering and the School of Design. The main learning objective is to formalize a methodical approach to needs assessment based on user-centered research. While in rotation in the clinical departments, student teams are matched with a clinical mentor who provides guidance and oversight. The clinical mentor in each of the hospital clinics oversees the students while in their respective rotation, addressing questions and providing clarification on procedures, norms, and general commentary regarding process. Mentors promote interaction between students, physicians, clinical staff and patients. The students are required to write twice weekly blog posts during their clinic rotations (read the blog entries on the CIP website: https://clinicalimmersion.uic.edu/). These posts serve as both a record and a reflection of their experience throughout the internship and serve as a group conversation starter during Monday morning workshops. Student teams are also required to write a report and create a presentation for each clinical rotation. The student teams present to their clinical mentor during the last week of the rotation. In addition, each team presents to the entire group of Clinical Immersion Program participants and faculty at the end of each rotation. 
In 2016 the program ran for six weeks through July and August and the following modifications were implemented:

- Program participants were expanded to include nine medical students from the UIC Innovation Medicine "IMED" program.

- Total number of participants in the 2016 program increased to 21 students (12 bioengineering students and 9 medical students). Participants rotated in teams of up to four in a mixed team of IMED and bioengineering students.

- The program remains a full-time paid internship for the bioengineering students. IMED students earn elective credit in their medical school curriculum.

- The following clinical departments participated in the program: Anesthesiology, Emergency Medicine, Gastroenterology/Hepatology, Hematology/Radiation Oncology, Neurology, Ophthalmology, Orthopedics, Radiology, Pulmonary Critical Care, Transplant Surgery and Urology

\section{$4 \quad$ Results and Discussion}

In the Clinical Immersion Program, bioengineering students and IMED medical students are teamed together to conduct a needs assessment in a clinical department. Some examples of the needs the student teams identified in clinical rotations are:

- Physical obstacles in the OR (Anesthesiology)

- Monitor sterility in the OR to lessen infection (Anesthesiology)

- Ergonomics of ophthalmoscope in order to avoid clinician neck and back strain (Ophthalmology)

- Accumulation of bacteria on underside of IV pumps (Pulmonology and Critical Care)

- Poor integration of technologies in OR (Pulmonology and Critical Care)

- Improve communication for patients on ventilators (Pulmonology and Critical Care)

- Lack of proper mobility of laparoscopic tools in the OR (Urology)

- Missing locks on supply cabinets (Emergency Department)

- Lack of routine check up of devices (Emergency Department)

- Gear failure in brachytherapy device (Hem/Onc and Rad Onc)

- Obtaining meaningful data from EEGs due to artifact (Neurology)

- Inability of 2D (planar) systems to navigate 3D tissue (Radiology)

- Inability of stents to respond to changes in flow or pressure (Radiology)

- Fistula infections in kidney dialysis patients (Transplant Surgery)

The following discussion relates to a survey that was administered in August 2016 to all 21 bioengineering and medical students who participated in the 2016 Clinical Immersion Program. In addition, a brief survey was emailed in January 2017 to previous CI Program participants (2014-2016) regarding the overall impression of the program and the impact on their work or current position. The survey was sent to 18 participants and 11 were returned (61\% response rate). (We were unable to establish contact with 2 past participants.) Their responses are incorporated into this discussion. 
The following table is a summary of the 11 former CIP participants who responded to the January 2017 survey and their current positions:

\begin{tabular}{|c|c|c|c|c|}
\hline $\begin{array}{c}\text { Graduate } \\
\text { school }\end{array}$ & $\begin{array}{c}\text { Medical } \\
\text { school }\end{array}$ & $\begin{array}{c}\text { Industry } \\
\text { Employment }\end{array}$ & Unemployed & $\begin{array}{c}\text { No } \\
\text { response }\end{array}$ \\
\hline 4 & 1 & 4 & 1 & 1 \\
\hline
\end{tabular}

Table 1: Past participants of Bioengineering Clinical Immersion Program and current employment/student status.

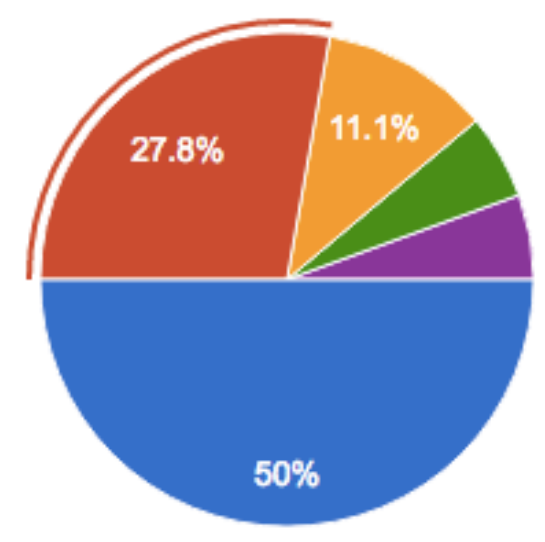

Strongly agree

Agree

Neutral

Disagree

Strongly disagree

Figure 1. Responses to "This internship helped me think differently about needs finding and problem identification” $(\mathrm{n}=18)$.

A primary outcome of this program is to adequately prepare students for the design of medical devices that are conceived from validated end-user needs. A core component is to introduce participants to the concept of user-centered design, where the design process is iterative and informed by a deep understanding of the user and environment of the product. Students are introduced to the methodology (specifically interview and observation techniques) to enable them to gain better understanding of users and stakeholders. Figure 1 illustrates the responses $(\mathrm{n}=18)$ to the statement "This internship helped me think differently about needs finding and problem identification." The majority of students strongly agreed or agreed with this statement. Further elaboration on this question supported the introduction of this material, as evidenced by the following responses: 
"The process of observing, assessing, and forming our thoughts into a coherent presentation was definitely helpful."

"Before it's so easy to see a problem, and instantly think of a solution. But this program made us look further into the problem and see why this problem is present."

"The program didn't change the way I think about needs finding and problem identification, but it did give me the chance to practice these skills more."

\section{Rate your experience working in an interdisciplinary team. (18 responses)}

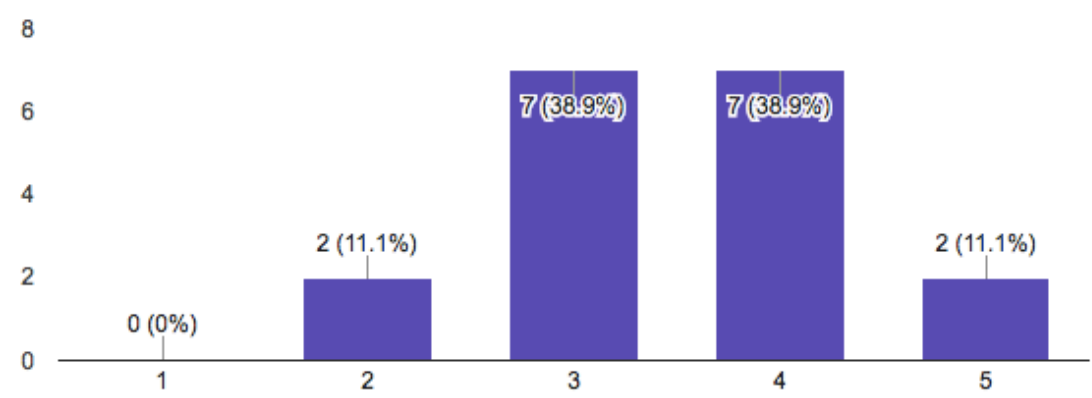

Figure 2. Responses to "Rate your experience working in an interdisciplinary team." (n=18) (1=least satisfactory and $5=$ highly satisfactory)

A key goal of the program is to improve students' interpersonal skills, collaboration and teamwork. Students were asked to rate their experience of working together on a team with both medical and bioengineering students. The majority (78\%) of students responded that the experience was satisfying or highly satisfying.

Adding medical students to the CI Program (several of whom either had an undergraduate degree in engineering or a strong interest in engineering) increased the team understanding of clinical procedures and current equipment/devices employed. It was unclear before the 2016 program began if this mix of bioengineering students and medical students would be productive, as there were notable differences in age, experience and goals. Both groups, however, seemed to benefit from the combined teams. The students worked well together in clinical rotations as evidenced by the high quality of team reports and presentations, as well as comments on the end of program survey:

"It was nice to have the medical students around to offer a medical perspective when we had questions. The interpersonal aspect was less than ideal at times, I'm not sure if this is due to the interdisciplinary aspect or not, but to be honest they talk a lot and it sort of gets in the way of progress sometimes."

"I very much enjoyed working with the engineering students. Especially as someone who does not have an engineering background I was able to learn a lot" 
Although 2016 program participants for the most part responded positively to the program and reported to have gained knowledge and experience from the internship, the majority of the comments to the question "What changes would you like to see in the program?" indicated a desire to move beyond needs finding and problem identification in order to delve deeper into the design process: specifically working on concepts and solutions to address those problems. Both medical students and bioengineering students were eager to brainstorm concept solutions to address problems they encountered in the rotations.

"I believe the main purpose of the summer program cannot be clinical immersion but rather trying to actually produce something of value and in the process learn the design process".

"While I understand the focus of the program was observation, I would have gained more had we learned more about what to do with those observations".

"I would like to add a hands on portion. I would reduce the amount of time spent in the clinic. I would encourage immediate research following identification of a problem".

This feedback about delving deeper into the design process was reinforced by former participants and their responses to the January 2017 survey. After 6 months to 1.5 years out of the program, most bioengineering participants still were pleased with their experience in the program. However the majority of responses to the question "What was lacking from the CIP program?" echoed the sentiment about moving beyond needs finding.

"It would have been nice to actually prototype an idea, even in the most basic form. That way we could see if what we thought could be a solution is actually viable".

“... actually proposing an idea to the hospital and making it happen”

"Find a way to incorporate emphasis on the finished product"

5.

Program Challenges and Modifications

Although the Clinical Immersion Program has run successfully since 2014, the program has evolved and expanded based on feedback from students and mentors in the program. Blog writing continues to be an issue for most students but this is one aspect of the program that has not changed despite student feedback that blog entries are tedious and taxing to write. The blogs serve as an individual reflection and requires students to put in the time twice weekly after hours to write about their experience. This is a requirement and a core component of the program and a learning tool. The co-instructors remind students to maintain the blogs and encourage them to use this as a tool for making sense of what they see in the clinic environment. Blog templates allow students to write and upload photos with minimal effort and not requiring web/blogging experience. In the upcoming 2017 program, there will be increased emphasis on assessing student blog posts to observe if students are applying what they learn in the workshop sessions to their clinical rotations. 
The most significant change planned for the Clinical Immersion Program 2017 based on student feedback is the expansion of the program to move beyond needs identification to include problem validation, evaluate current alternates and brainstorm concept solutions. The Monday morning sessions will be expanded to full day $(9 \mathrm{am}-3 \mathrm{pm})$ workshops to allow for greater opportunities to guide students through the process. In previous years, there was not a perceived improvement of the students to identify needs from the first rotation to the second, perhaps due to a steep learning curve of the differences between clinical rotations. In 2017, student teams ( 2 bioengineering students plus 2 medical students) spend the full duration of the program in a single clinical department, thereby allowing for a deeper dive into the clinical environment. This will enable teams to identify a problem and propose a concept that may address the problem as well as time to prototype and test their concept. This task will challenge the students and will require a rigorous schedule. The goal is to gain deeper experience in validating the identified needs by evaluating the current alternatives address the need, and brainstorming concept solutions. We recognize the challenge in compressing the process into a six-week experience, but believe having students validating their problem in this way will better prepare them for their senior design capstone course. While it is not expected that the teams will produce physical prototypes, the emphasis will be on concept sketches and competitive analysis of current alternatives. IMED students also participate in the Interdisciplinary Medical Product Development course in the fall, an option for bioengineering students as their design capstone course.

In past years getting feedback from clinical mentors has been a challenge. Effort was made at the conclusion of each Clinical Immersion Program to solicit responses from clinical professionals but the communication has been difficult and often incomplete. Moving forward in the 2017 program, quantitative measures will be put in place to help assess satisfaction, and evaluate the program, both from the student and mentor perspective. For example, written guidelines will be supplied for clinical mentors including goals of the program and expected outcomes in order to enhance student engagement before the program begins. These guidelines can then be presented back to students and mentors at the end of the internship in order to evaluate the internship experience. Documentation tools and techniques (such as interviews, observation, prototyping and testing) can also be measured at the end of the 6 -week internship).

\section{Conclusion}

There is a need to close the gap between academic programs and the design of medical devices based on validated user needs. The Clinical Immersion program has been a bridge for bioengineering and medical students to apply their academic knowledge and practice the design process. The focus of the program has been to expose students to the user-centered design process and prepare them for the Senior Design course sequence. Students report better understanding of user-based design and the needs identification in the clinical environment. In addition, working in interdisciplinary teams of engineering and medical students was a valuable, positive experience. Based on the success of the first three years of the program and feedback from former participants, we plan to expand the program in 2017 to include more of the design process in order to guide students through needs validation, current alternative solutions and concept generation. Students will work on a team consisting of both bioengineering and medical students in a clinical department for six weeks, allowing for a deep dive to identify problems and possible 
opportunities. They will evaluate possible concepts and prototype and test those ideas in the clinical environment. This opportunity is essential for students interested in medical device design. User-centered design instruction will be expanded in the Monday all day session, providing students with a rich humancentered toolkit for a deep needs assessment, a set of tools for creative ideation, and a comprehensive qualitative toolkit for iterative phases of rapid improvement. It is exciting to grow the program in order to continue to meet the needs of both bioengineering and medical students.

\section{Acknowledgements}

Research reported in this publication was supported by National Institute of Biomedical Imaging and Bioengineering of the National Institutes of Health under award number R25EB018239. The content is solely the responsibility of the author and does not necessarily represent the official views of the National Institutes of Health.

\section{8. $\quad$ References}

[1] M Kotche. Clinical Immersion Internship Introduces Students to Needs Assessment. Paper presented at American Society of Engineering Education, New Orleans, LA, June 2016. 10.18260/p.26503

[2] Zoltowski, Carla B., William C. Oakes, and Monica E. Cardella. "Students' Ways of Experiencing Human-Centered Design,” Journal of Engineering Education,” 101(1), 28-59, 2012.

[3] Privitera, Mary Beth, M. Design and Dale, L. Murray. "Applied Ergonomics: Determining User Needs in Medical Device Design," in 31st Annual International Conference of the IEEE EMBS, Minneapolis, MN, 2009.

[4] Gertner, Michael. "Biomedical Innovation, Surgical Innovation, and Beyond," in 9th Annual National Collegiate Inventors and Innovators, San Diego, CA, 2005.

[5] Walker, M. \& Churchwell, A.L. "Clinical Immersion and Biomedical Engineering Design Education: Engineering Grand Rounds.” Cardiovasc Eng Tech (2016) 7: 1. doi:10.1007/s13239-016-0257-y

[6] Stephens, J. S., \& Rooney, S. I., \& Arch, E. S., \& Higginson, J. (2016, June), "Bridging Courses: Unmet Clinical Needs to Capstone Design (Work in Progress)," Paper presented at 2016 ASEE Annual Conference \& Exposition, New Orleans, Louisiana. 10.18260/p.26393

[7] Przestrzelski, B., \& DesJardins, J. D., \& Brewer, C. M. I. (2016, June), "Year Two - The DeFINE Program: A Clinical and Technology Transfer Immersion Program for Biomedical Needs Identification and Valuation," Paper presented at 2016 ASEE Annual Conference \& Exposition, New Orleans, Louisiana. 10.18260/p.27062

[8] Kadlowec, J., \& Merrill, T., \& Hirsh, R. A., \& Sood, S. (2015, June), "Work-In-Progress: Clinical Immersion and Team-Based Engineering Design," Paper presented at 2015 ASEE Annual Conference \& Exposition, Seattle, Washington. 10.18260/p.25098 
[9] Soh, E. K., \& Kaur, A., \& Tham, M. P., \& Chong, D. Y. (2013, June), "Engineers in Hospital: An Immersive and Multidisciplinary Pedagogical Approach for Better Solutions," Paper presented at 2013 ASEE Annual Conference \& Exposition, Atlanta, Georgia. https://peer.asee.org/19541

[10] Wright, T., \& Myers, E., \& Bartel, D., \& van der Meulen, M. (2001, June), “An Immersion Term In Biomedical Mechanics" Paper presented at 2001 Annual Conference, Albuquerque, New Mexico.

https://peer.asee.org/9340 